Gerión. Revista de Historia Antigua

ISSN: 0213-0181

http://dx.doi.org/10.5209/GERI.56966

\title{
El Ottaviano Capoparte de Mario Attilio Levi y su influencia en The Roman Revolution de Ronald Syme
}

\author{
Gustavo Alberto Vivas García ${ }^{1}$
}

Recibido: 13 de enero de 2017 / Aceptado: 5 de mayo de 2017

Resumen. El Ottaviano Capoparte, la monografía en dos volúmenes escrita por el historiador italiano Mario Attilio Levi (1902-1988), publicada en Florencia en 1933, ejerció una gran influencia en el proceso de composición de The Roman Revolution (1939), la obra más conocida de Ronald Syme (1903-1989). La obra del italiano se cita hasta en diez ocasiones en La revolución romana, lo que da una idea de la importancia que la obra de Levi tenía para el especialista neozelandés. Términos como "partido", "facción" y "Estado Nuevo", que aparecen de manera recurrente en Ottaviano Capoparte, están presentes a lo largo de todo el libro de Syme y en nuestro artículo queremos demostrar que éste tenía muy presente, quizá en su mesa de trabajo, la obra de Levi cuando escribía La Revolución Romana.

Palabras clave: Octaviano; partido; facción; Historia de Roma.

\section{[en] Mario Attilio Levi's Ottaviano Capoparte and its influence on Ronald Syme's The Roman Revolution}

Abstract. The Ottaviano Capoparte, the two-volume monograph written by the Italian historian Mario Attilio Levi (1902-1988), published in Florence in 1933, exerted a great influence on the process of composition of The Roman Revolution (1939), the best-known work by Ronald Syme (1903-1989). The work of the Italian is quoted ten times in The Roman Revolution. This fact gives an idea of the importance that the work of Levi had for Syme. Terms such as "party", "faction" and "new state", which recurrently appear in Ottaviano Capoparte, are present throughout Syme's book. In our article we want to show that Syme had very present, perhaps at his working table, the monograph by Levi when he wrote The Roman Revolution.

Keywords: Octavian; Party; Faction; History of Rome.

Sumario. 1. Apuntes introductorios y biográficos. 2. El Ottaviano Capoparte y The Roman Revolution. 3. La influencia de $O C$ en $R R$ en el planteamiento de los hechos históricos y en la bibliografía. 4. La influencia de $O C$ en $R R$ en el uso de la terminología política. 5. Conclusión. 6. Referencias bibliográficas.

Cómo citar: Vivas García, G. A. (2017) El Ottaviano Capoparte de Mario Atilio Levi y su influencia en The Roman Revolution de Ronald Syme, en Gerión 35/1, 277-295.

\footnotetext{
Universidad de La Laguna

E-mail: gusgarvi@gmail.com
} 


\section{Apuntes introductorios y biográficos ${ }^{2}$}

Uno de los principales objetivos de Ronald Syme cuando escribió The Roman Revolution [en adelante $R R$ ] era escribir un gran lienzo narrativo del final de la República romana y el advenimiento del Principado. De todos es sabido que, a la hora de alumbrar una obra tan importante, la influencia en Syme, tanto de Matthias Gelzer y su Die Nobilität der römischen Republik (1912) como de Friedrich Münzer con su Römische Adelsparteien und Adelsfamilien (1920), resultaron decisivas. Un Syme por cierto que, en contra de la idea de Momigliano, ${ }^{3}$ nunca reconoció haber leído a Namier y su The Structure of Politics at the Accession of George III (1929).

Menos conocida es, sin embargo, la influencia que en la concepción de $R R$ tuvieron los trabajos de Richard Heinze (1867-1929), Catedrático de Historia Antigua en Leipzig de 1906 a 1929, sobre conceptos como fides, pietas o auctoritas recopilados en su obra Vom Geist des Römertums (1938). La obra de Heinze ejerció una influencia enorme en Gelzer y en Syme, aunque este influjo haya pasado inadvertido para buena parte de los especialistas. ${ }^{4}$ Los temas de trabajo de Heinze eran los nobiles y las redes clientelares y, sin duda, Syme conocía y había leído buena parte de la bibliografía de este erudito alemán.

En este trabajo nos ocuparemos de otra de las grandes influencias de Syme a la hora de idear y escribir La Revolución Romana: los dos volúmenes publicados por el italiano Mario Attilio Levi en 1933 con el llamativo título de Ottaviano Capoparte.

Al historiador de la Antigüedad Mario Attilio Levi le fue concedido el aparente don de una larga existencia. Nacido en Turín en 1902 y fallecido en 1998 en Pregassona, en la actualidad un barrio de la ciudad suiza de Lugano, su vida corre paralela a la del convulso siglo veinte. Su dilatada trayectoria personal estuvo relacionada, además, con alguno de los hechos más oscuros y controvertidos de la historia del pasado siglo: el establecimiento del fascismo en Italia y la Segunda Guerra Mundial. ${ }^{5}$

Al comienzo de su carrera, Levi fue profesor de historia romana y griega en

2 Agradezco a los profesores José A. Delgado Delgado (La Laguna) y Anthony R. Birley (Düsseldorf) la ayuda que me han proporcionado durante la redacción de este trabajo. Ambos han leído el original y me han aportado valiosísimas sugerencias. El Prof. Daniele Foraboschi (Milán), leyó también el original del texto y me proporcionó atinadas indicaciones. Alan Brown (Biblioteca Bodleiana, Oxford), tuvo la amabilidad de facilitarme un importante documento. El Prof. Fernando Wulff (Málaga), compartió conmigo un perspicaz trabajo sobre este asunto que, hasta donde conozco, permanece inédito. Los dos evaluadores anónimos me han proporcionado ideas muy útiles. Los años correspondientes a los hechos históricos que se narran en el artículo son antes de Cristo a menos que se indique lo contrario. Cualquier error en la versión final del texto es de mi responsabilidad.

3 Momigliano 1962, x $(=1966,730)$. Momigliano escribe en su Introducción que Syme ha "namierizzato la costituzione di Augusto" (loc. cit.). A favor de esta opinión, vid. AlföLdy 1983, 10-11 donde el sabio alemán de origen húngaro piensa que Momigliano da en el clavo cuando resalta la influencia de Namier y de su atmosfera intellettuale en Syme y su conocida obra. Contra BIRLEY 2016, 159 y en otros lugares: vid. por ejemplo VIVAS García 2016, 39. Birley piensa que esta idea carece de fundamento y que hemos de creer a Syme cuando asegura que no leyó la obra de Namier antes de escribir $R R$. Este texto de Momigliano de 1962 es importante para nuestro trabajo por cuanto comenta no solo las distintas contribuciones de Levi, como la que analizamos, sino también su Il tempo di Augusto (Levi 1951), que es una puesta al día de los dos volúmenes de 1933. Por supuesto, este es un camino que Momigliano comenzó a roturar con su conocida reseña: MomigLIANO 1940, 75-80.

$4 \quad$ No así a Sir Fergus Millar que la pondera en su justa medida en MiLlar 1981, 145.

5 Para unos apuntes biográficos sobre la figura de Levi debe consultarse sobre todo Musti 1993. Puede complementarse con el propio LeVI 1989, Polverini 2006 y MurRaY 2010. Y por supuesto con GARA FORABOSCHI 1993, un texto fundamental para entender la trayectoria de nuestro autor. 
la universidad de Turín. Desde 1936 fue profesor de historia romana en la universidad de Milán y de historia antigua de Grecia en la misma universidad de 1968 a 1977. Fue socio correspondiente de la Accademia dei Lincei desde 1988 y elegido socio nacional de la misma institución un año después. Durante los años de persecución antisemita en Italia, a raíz de las leyes promulgadas en 1938 por el gobierno fascista, tuvo que publicar, bajo el pseudónimo de Manlio Canavesi, obras como La politica estera di Roma antica $(1942)^{6}$ o Nerone: Saggio storico (1945), ${ }^{7}$ reelaborado y reeditado después con su verdadero nombre y bajo el título Nerone e i suoi tempi en 1949. ${ }^{8}$ Levi fue, por cierto, el autor junto con G. Funaioli y G. Cardinali de la voz "Cesare, Gaio Iulio" para la Enciclopedia Italiana en 1931, artículo que no fue en absoluto del gusto de Mussolini. ${ }^{9}$

Levi participó de manera activa como combatiente en la Segunda Guerra Mundial. En septiembre de 1943 ganó una medalla de plata al valor por su acción de resistencia ante las fuerzas de ocupación alemanas en la Puerta de San Paolo (Roma). Un año y medio después, como teniente de los alpini del Grupo de Combate "Friuli", participó en la liberación de la localidad de Imola el 14 de abril de $1945 .{ }^{10}$ Después de la guerra, en 1947, prestó testimonio a favor de Cesare Maria de Vecchi, conde de Val Cismon (1884-1959), que fue gobernador fascista de la Somalia italiana de 1923 a $1928 .{ }^{11}$

Tras su retorno a la universidad, después de haber sido apartado de la misma en 1938, se dedicó al estudio de diversos aspectos de la historiografía y la cultura política de la antigua Grecia, en especial desde mediados de los años 50 del siglo pasado. La figura académica de Levi y su influencia en el mundo universitario italiano fue enorme. Fue profesor invitado en varias universidades norteamericanas, Director del Centro Studi e Documentazione sull'Italia romana y Presidente del Comité Internacional para el Estudio de la Ciudad Antigua, con sede en Estrasburgo. Actualmente, y como homenaje a su persona y su trayectoria, uno de los auditorios de la universidad de Milán lleva su nombre.

Milano, Istituto per gli studi di politica internazionale, 1942.

Milano, Messina. Giuseppe Principato, 1945. Hay una edición anterior hecha en Milán en 1943, según Musti. No he podido localizar la editorial de este título.

Milano, Istituto editoriale Cisalpino, 1949.

9 Cf. Cannistraro 1975, 371, n. 129.

10 Véase la siguiente dirección de internet: www.noialpini.it/levi-mario-attilio.htm (último acceso el 12 de octubre de 2016).

11 Sobre el personaje vid. SANTARELLI 1991. 
A día de hoy, la figura de Levi sigue planteando interrogantes a los especialistas. El perspicaz Giusto Traina lo ha descrito recientemente como un "fascista "di sinistra". ${ }^{12}$ Arnaldo Marcone lo ha tildado de "fascista eterodosso". ${ }^{13}$ Era de ascendencia judía y, sin embargo, fue un activo miembro del partido fascista a pesar de que tuvo que dejar su puesto en la universidad de Milán debido a la promulgación de las leyes de 1938 y verse obligado a escribir bajo seudónimo, como hemos visto. De hecho, hacia el final de su vida él mismo escribió lo siguiente a modo de explícita confesión:

Queste contrapposizioni politiche non avevano echo nella vita universitaria del secondo decenio del secolo [XX], anche se De Sanctis [su maestro y mentor Gaetano De Sanctis (1870-1957), que fue también maestro de Arnaldo Momigliano (1908-1987)] e tutti i suoi allievi, a eccezione di chi scrive, avevano assunte posizioni ostili al fascismo in maniera generalmente non attiva, mentre L. Pareti [(1885-1962), también discípulo de De Sanctis, como los dos autores anteriores] aveva aderito al fascismo sin dall' inizio. ${ }^{14}$

Parece fuera de duda el hecho de que Levi, que provenía de una familia de raíces judías igual que Momigliano, ${ }^{15}$ se adhirió al movimiento fascista desde los comienzos del mismo. El mismo Momigliano fue también miembro del Partido Fascista, y además un miembro muy activo. ${ }^{16}$

\section{El Ottaviano Capoparte y The Roman Revolution}

Buena parte de la producción de Levi durante su larga vida se consagró al estudio de la historia romana. En este trabajo nos centraremos en una obra en dos volúmenes que publicó en 1933: Ottaviano Capoparte. Storia politica di Roma durante le ultime lotte di supremazia. ${ }^{17} \mathrm{El}$ historiador italiano publicó de nuevo ambos volúmenes en 1951 con añadidos y correcciones bajo el título Il tempo di Augusto,${ }^{18}$ y finalmente en 1986 y 1994 como Augusto e il suo tempo. ${ }^{19}$ En 1933, cuando se publicó el libro, Levi ejercía su magisterio en Turín, donde era uno de los discípulos del afamado profesor Gaetano de Sanctis. ${ }^{20}$

En este artículo queremos demostrar la importancia que esta obra tuvo para Syme (1903-1989). ${ }^{21}$ Éste conoció la obra de Levi desde el momento en que ésta vio la luz,

12 Traina 2014, xiv.

13 Marcone 2015, 626. Lo heterodoxo de la ideología fascista de Levi no se percibe en ningún momento en $O C$. Esto permitió a Syme alinearse con la obra del italiano. Además en 1933, cuando Levi escribió su obra, el consenso general en Italia sobre Mussolini no era en absoluto negativo por lo que muchos académicos de prestigio en el país eran miembros del partido fascista, caso de Levi y del propio Momigliano.

14 LEVI 1989, 10.

15 Por cierto, sobre Momigliano y Syme y sobre la relación entre ambos sabios se han escrito ríos de tinta. La que quizá sea la última aportación sobre el tema la ofrece BIRLEY 2016.

16 Véase Di Donato 1995, 213 y ss. Aunque en su exilio en Inglaterra, se convirtió en un furibundo activista antifascista.

17 Editado por "La nuova Italia” Editrice en dos volúmenes (LEVI 1933).

18 Firenze, "La nuova Italia" Editrice, 1951.

19 Milano, Rusconi, 1986 y 1994.

$20 \quad$ Vid. LEVI 1989.

21 Sobre la figura de Syme puede consultarse: ALFÖLDY 1983; BowersocK 1980, 1991, 1994 y 1995; MILLAR 1981; Birley 1989; Brunt 1989 y 1996; Devine 1989a y 1989b; Christ 1990; Galsterer 1990; GrifFin 
como lo demuestra el hecho de que la reseñó para la revista The Classical Review en un texto que apareció en 1934. ${ }^{22}$ En nuestra opinión, reseñar a Levi y el hecho de que conociera en profundidad la monografía del italiano, supuso para Syme un momento crucial en su desarrollo como historiador.

El propio Syme decidió situar esta reseña como el primer trabajo que figura en el sexto volumen de sus Roman Papers, ${ }^{23}$ un claro indicio de la importancia que tenía para él. En este sexto volumen de sus trabajos, Syme quiso incluir una serie de textos que Badian no había incorporado en los dos primeros volúmenes editados por él de los Roman Papers,${ }^{24}$ caso de esta reseña, en cuyo proceso de redacción y preparación Syme trabajó intensamente. ${ }^{25}$

La obra de Levi supuso para Syme un aldabonazo y la influencia de la obra del historiador italiano fue crucial en el proceso de escritura, por parte del neozelandés, del libro que comenzaría a escribir tres años más tarde, en el verano de 1936, y que se convirtió en su obra más famosa y conocida: The Roman Revolution, publicada por la Oxford University Press el 7 de septiembre de 1939.

Nuestra idea es que Syme tuvo bien presente los dos volúmenes del Ottaviano Capoparte [en adelante $O C$ ], mientras escribía su magnum opus. Esta influencia, que a nuestro modo de ver es clara y duradera, ha pasado inadvertida para la mayoría de los investigadores durante los casi ochenta años que han transcurrido desde la publicación de ambas obras.

Levi dedicó los dos libros que componen $O C^{26}$ a analizar de manera pormenorizada la labor de Octaviano durante los años que discurren entre el año 44 y Accio (31). No obstante, $O C$ es una obra que va más allá de su propósito inicial. Es un fresco histórico del final de la República romana por el que transitan personajes como Cicerón, Marco Antonio, Fulvia, Sexto Pompeyo o el propio Octaviano.

El $O C$ llega a citarse hasta en un total de diez ocasiones en The Roman Revolution: 115 y ss., n. $4[157]^{27}$ (ref. 1); 191, n. 4 [248, n. 21] (ref. 2); 225, n. 2 y $3[289$, n. 53 y 54$]$ (refs. 3 y 4$) ; 260$, n. 1 [330, n.7] (ref.,5); 265, n. 3 [336, n. 27] (ref. 6); 273, n. 2 [346, n. 55] (ref. 7); 274, n. 2 [346, n. 58] (ref. 8); 280, n. 3 [355, n. 21] (ref. 9); 290, n. $1[366$, n. 58] (ref. 10). Esta mera enumeración da una idea, en nuestra opinión, de la importancia que Syme otorgó a la monografía de Levi, toda vez que las notas de bibliografía secundaria son más bien escasas en $R R$.

Una simple lectura de $R R$ permite observar el hecho de que, de las diez citas, seis se encuentran en las 34 páginas que componen los capítulos 19 y 20 de la obra: los titulados "Antonius in the East" y "Tota Italia", respectivamente. Lo que creemos

1990; Alonso Troncoso 1990; Arce Martínez 1990 y 1994; Caballos Rufino 1993 y Wiseman 1998. Más recientemente puede consultarse a Walter 2002; Dahlheim 2003; Devine 2004 y Toher 2009, aunque en este artículo se hace tan sólo un breve bosquejo biográfico de Syme; así como a ArCE Martínez 2010; Matthews 2010 y ReBEnICH 2014. Las últimas investigaciones sobre el personaje son las de Vivas García 2016 y SANTANGELO 2016.

22 SyMe 1934, 76-78.

23 SYME 1991.

24 SYME 1979

25 El propio Syme le confesó a Birley este particular poco antes de su muerte en una de las reuniones que mantuvieron para determinar qué trabajos se incluirían en el volumen seis de los Roman Papers.

26 El primero de los volúmenes tiene 264 páginas y el segundo 277 , respectivamente, incluyendo este último un índice de nombres.

27 A lo largo de todo el artículo figuran entre corchetes las referencias a la primera edición en castellano de $L a$ Revolución Romana: Syme 1989. 


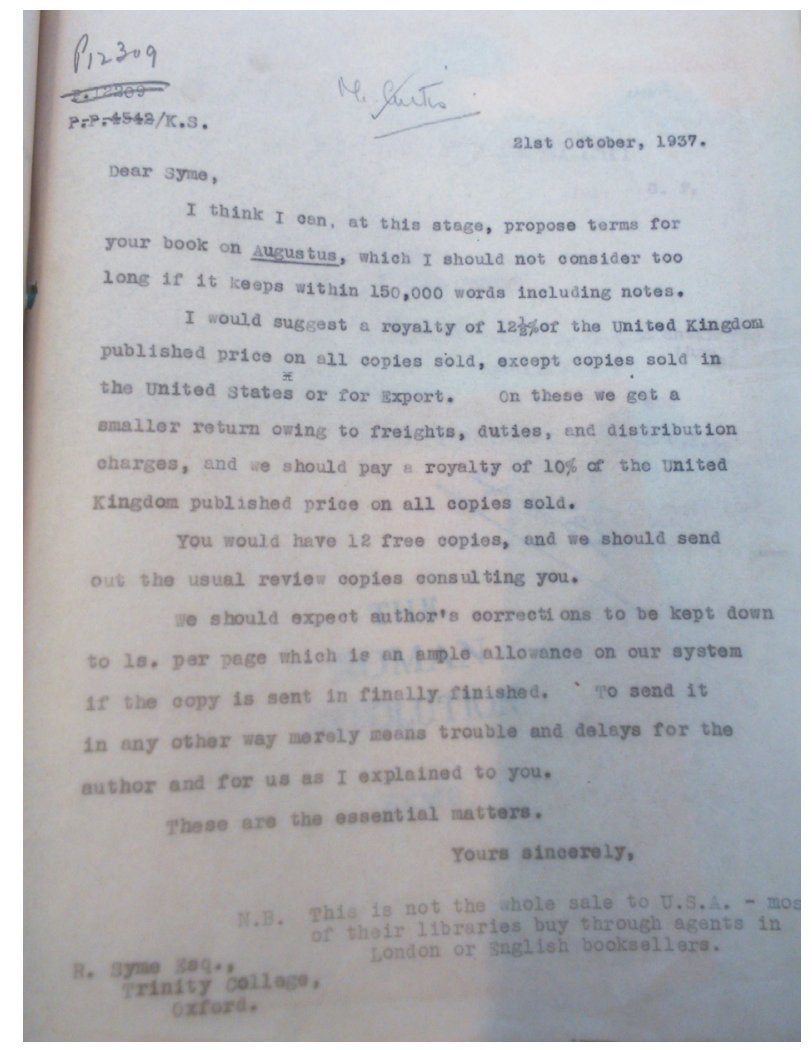

Figura 2. Carta de Kenneth Sisam a Ronald Syme. 21.10.1937. Archivo editorial BACK B00551 (Oxford University Press).

que refuerza nuestra tesis de que Syme usó generosamente la monografía de Levi, más en concreto, en la redacción de ambos capítulos. Sabemos que, en el verano de 1937, Syme tenía redactado un primer borrador completo de su libro hasta, por lo menos, ese capítulo $20 .{ }^{28}$

Todo ello, sin mencionar el importantísimo hecho de que, al menos desde finales de octubre de $1937^{29}$ (Fig. 2) hasta finales de enero de $1939^{30}$ (Fig. 3), The Roman Revolution iba a llamarse "Augustus: Leader and Party", que es el nombre que lleva por título el archivo editorial de $R R$ en la Oxford University Press. ${ }^{31}$

28 TOHER 2009, 324.

29 Carta de Kenneth Sisam (ejecutivo de la Oxford University Press) a Syme de 21 de octubre de 1937 que figura en el archivo editorial BACK B00551 de la Oxford University Press. El archivo lleva por título "Augustus: Leader and Party". Agradezco la ayuda prestada por el Dr. Martin Maw, archivero jefe de la Oxford University Press, y por Beverley McCulloch, archivera de la editorial, en la consulta de este material durante mi estancia en Oxford en julio de 2016. Los documentos mencionados en esta nota y la siguiente se reproducen en este trabajo con el permiso expreso de la Oxford University Press, poseedora del copyright de los mismos.

30 Orden de publicidad sobre The Roman Revolution de fecha 26 de enero de 1939. Archivo editorial BACK B00551. Oxford University Press.

31 Este hecho, relativamente poco conocido, ha sido puesto de manifiesto por el profesor Christopher Pelling en la Syme Lecture que pronunció en Wolfson College el 6 de noviembre de 2014, publicada después en forma de 


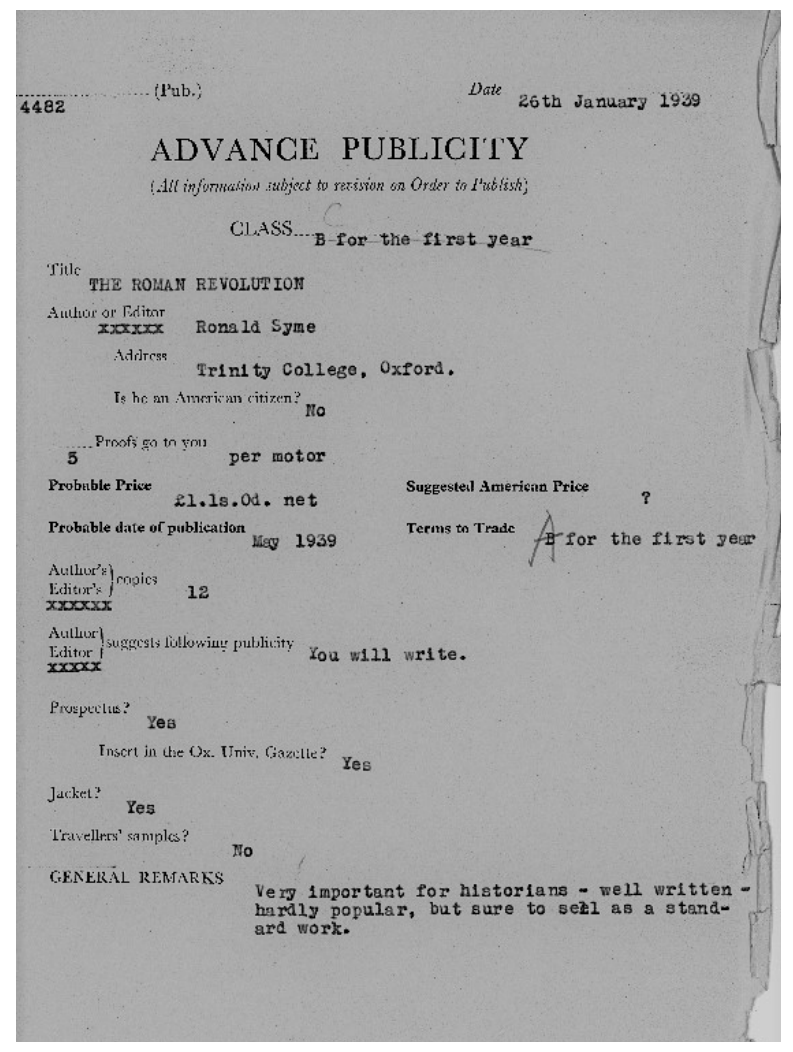

Figura 3. Orden de publicidad de The Roman Revolution. 26.01.1939 Archivo editorial BACK B00551 (Oxford University Press).

A este respecto, es fundamental mencionar que en la Biblioteca Bodleiana se encuentra el manuscrito original de The Roman Revolution, manuscrito que lleva por título asimismo "Augustus: Leader and Party". ${ }^{32}$ A escasos meses de que el libro viera la luz, el cambio de título a La revolución romana le fue sugerido a Syme desde la órbita de la editorial. No sabemos exactamente por quién. ${ }^{33}$ El manuscrito de la Bodleiana parece una penúltima versión de lo que Syme entregó a la imprenta y data probablemente de 1937 o comienzos de 1938. Es un documento fascinante y cualquier lector enterado habrá concluido, a estas alturas de nuestro razonamiento, que "Augustus: Leader and Party" es la traducción, no exacta eso sí, del italiano al inglés del título de la monografía de Levi. ${ }^{34}$

Sobre el asunto de la elección final de los términos Roman Revolution para el título de su monografía, en una reseña publicada en 1937 sobre dos obras dedicadas

artículo: PeLLing 2015, 207-247.

32 Bodleian MS Eng. d. 2099. Este artículo debe considerarse un trabajo preparatorio, o Vorarbeit, del proyecto que tenemos entre manos y que cuenta con el permiso y la anuencia del Prof. Sir Fergus Millar, albacea literario de Syme: la edición por nuestra parte de este manuscrito de casi 900 páginas.

33 Cf. n. 36. Kenneth Sisam muy bien pudo estar detrás de esta decisión del cambio de título.

34 Pelling 2015, 212. 
al análisis de las figuras de Augusto y de su socio Agripa, Syme escribió:

(...) it should help to reveal the Roman Revolution, the triumph of the faction of Augustus and the establishment of the Principate as something personal, immediate and tangible. ${ }^{35}$

Que sepamos, es la primera vez que en un texto Syme emplea el doble término "Roman Revolution", que será el título de su célebre monografía a la vuelta de un par de años. Un año después, escribe que la creación del concepto de "tota Italia":

(...) was not the product of abstract speculation applied to human affairs, but the work of time and circumstance, violently accelerated by Civil War and confiscation, by Dictatorship, and by Revolution. ${ }^{36}$

\section{La influencia de $O C$ en $R R$ en el planteamiento de los hechos históricos y en la bibliografía}

Uno de los aspectos en los que más se aprecia la influencia de $O C$ en $R R$ es en el análisis de los hechos históricos correspondientes al período triunviral, tratados en ambas obras. En bastantes ocasiones, Syme usa los mismos materiales que Levi utilizó en su obra tres años antes. Este uso de la misma bibliografía no es un hecho aislado, sino que es sintomático de la influencia y los íntimos lazos de unión entre ambas monografías.

Así, la narración (ref. 1) de cómo tres meses después de la muerte de Julio César, el 1 o 3 de junio de 44, Antonio consigue que el Senado vote la Lex de permutatione provinciarum. ${ }^{37}$

El final de la n. 4 de $R R$ en la página 116 de la edición inglesa, donde se menciona específicamente el primero de los volúmenes de $O C$, no figura completa ni en la traducción española de 1989 (157, n. 17); ni en la edición realizada en 2010 (149, n. 17). ${ }^{38}$ En el primer caso seguramente por un error del traductor, el Profesor Blanco Freijeiro y, en el segundo, por haberse basado en la traducción de 1989 y no haber consultado el original inglés, con lo que el error se perpetuó.

En el original inglés, la nota de Syme menciona el libro de T. R. E. Holmes The Architect of the Roman Empire, ${ }^{39}$ que también es mencionado por Levi en $O C(1,77)$. Es el uso de los mismos materiales en ambos autores al que aludíamos más arriba. Nuestros dos investigadores conocían bien el libro de Holmes (1855-1933), pero

\footnotetext{
SYME 1937, 194-195.

36 SYME, 1938, 31. Cf. AlFöLdy 2015, 207: "In the papers of Alföldi I have never come across the term 'Weltkrise'. The reason why he called his collected studies on third-century topics Studien zur Geschichte der Weltkrise des 3. Jahrhunderts nach Christus [Darmstadt, 1967], was, in my view, just to give the book an attractive title. We can recall that something similar was the case with The Roman Revolution of Ronald Syme, whose 'concept of revolution' was frequently criticised, above all by German scholars. I was once told by Sir Ronald that he had never had a 'concept of revolution'; the title of his famous book was proposed by his publisher as an attractive one, which the author liked 'because of the alliteration' (Ronald, Roman, Revolution)". Cf. también U. Kahrstedt, 'Die Grundlagen und Voraussetzungen der römischen Revolution', Neue Wege der Antike 4, 1927, 97-118, citado por Momigliano en su reseña de JRS de 1940, 78.

37 Cf. Rotondi 1912, 432.

38 SYME 2010.

39 Holmes 1928.
} 
es probable que tanto Levi como Syme consideraran que la visión que este autor proporciona de Augusto en su monografía era, como mínimo, un tanto complaciente, por no decir claramente indulgente. En el caso de Syme, al menos, y tras leer con atención $R R$ podemos colegir que la visión de Holmes sobre Octaviano le pareció poco atinada. La monografía publicada por Holmes en 1928 era claramente el trabajo de un anciano cuya perspicacia histórica comenzaba a fallar, falto del alcance y del rigor de trabajos anteriores suyos, como The Roman Republic, ${ }^{40}$ en el que ofrecía la misma visión amable, pero en este caso de Julio César.

El trato que Syme da en $R R$ a la monografía de Holmes de 1928 es benigno, quizá porque admiraba la minuciosidad de aportaciones anteriores del autor, especialmente en cuestiones topográficas a las que Syme siempre prestaba especial atención. ${ }^{41}$

La ref. 2 se centra en un espinoso episodio acontecido a finales de 43 y buena parte del año siguiente. La República asiste en este tiempo a un auténtico "reinado del terror". El funesto capítulo de las duras medidas triunvirales que conocemos bajo el nombre genérico de "proscripciones" y que supuso un verdadero golpe de gracia para la forma de gobierno republicana, ahondando de forma definitiva e irreparable en lo que a la postre resultó la muerte anunciada de la República.

Los efectos de las devastadoras medidas de los triunviros se dejaron sentir en especial entre las élites de la sociedad romana. Se ha hablado de aproximadamente 300 senadores y 2.000 caballeros ejecutados durante ese par de años ${ }^{42}$ en los que los triunviros, cual "vero "monstro a tre teste" en la afortunada expresión de Levi (OC $1,231){ }^{43}$ confiscaron a esos grupos dirigentes cuantiosos bienes, impulsados por la arbitrariedad y el deseo de revancha política.

Los triunviros actuaron de pleno acuerdo y, lo que al principio fue una onerosa medida económica de exacción o carácter impositivo, degeneró en un baño de sangre que sirvió para eliminar físicamente a cualquier oposición rica o influyente.

Es interesante el tratamiento que Levi da al asunto de las proscripciones y que Syme percibió bien cuando escribió que el autor italiano enfatizaba "perhaps too much the impersonal character of the proscriptions" (RR 191, n. 4 [248, n. 21]). Levi es comprensivo e indulgente hacia las figuras de los triunviros, y llama la atención que proporcione una justificación a la violencia inherente a las proscripciones, calificándola como algo provisional y necesario, ${ }^{44}$ como un paréntesis casi preparatorio de cara a la implementación de un régimen posterior que será distinto al existente, y no como lo que en realidad fue: un hecho de enorme violencia como base fundacional de un régimen cruento e inestable que duró una quincena de años hasta dar paso al gobierno monárquico del miembro más joven del triunvirato.

Esa justificación se palpaba en el ambiente y la época en la que Levi escribió su obra, aunque mucho de lo escrito por Levi en $O C$ "would not have been music to Mussolini's ears". ${ }^{45}$ A historiadores como Pelling ${ }^{46}$ les extraña que en $R R$, que puede sintetizarse como un declarado intento de desmontar el consenso positivo que se tenía sobre Augusto y su labor de gobierno en los años 30 del pasado siglo XX, Syme

\footnotetext{
HOLMES 1923.

Vid. Vivas García 2016, 31.

Bravo 1989, 142. Y, sobre todo, Hinard 1985. App. BC 5.5 proporciona estas cifras.

Vid. sobre la feliz expresión: GARA - ForABOsCHI 1993, 7 y ss.

En palabras de Levi: "una funzione senza dubbio limitata e provvisoria" $(O C 1,232)$.

Pelling 2015, n. 10.
}

46 Ibid. 
alabara explícitamente el tratamiento de Levi sobre este particular ${ }^{47}$ y casi exonerara a Octaviano de cualquier culpa. Por supuesto, después de referirse al juicio de Levi sobre las proscripciones, Syme criticó los comentarios de Rice Holmes sobre el mismo tema en su conocida obra:

(...) unfortunately marred by a piece of rank pragmatism. 'To support his colleagues was a part of the price which he had to pay for attaining the power that enabled him to become one of the greatest benefactors of mankind.' (The Architect of the Roman Empire i, p. 71). ${ }^{48}$

Los dos textos de esta referencia poseen resonancias más que ocasionales. Así, cuando Syme escribe la frase: "Their victory was the victory of a party" ( $R R 191$ [248]), la misma está remitiendo de manera inequívoca a otra escrita tres años antes por Levi en su monografía: "È un partito che conquista lo stato e aspira al dominio stabile" (OC 1, 230).

Los hechos hablan por sí solos. Estas similitudes, y otras que analizaremos en el siguiente apartado, demuestran que Syme tenía muy presente la monografía de Levi mientras escribía su $R R$, y que la obra del italiano influyó de forma importante en la escritura y la concepción de la obra del neozelandés.

En este apartado de la gran influencia de los materiales usados por Levi en Syme puede aducirse otro ejemplo más (ref. 3). En este caso, el trabajo de F. Blumenthal, que Syme cita en la página 225, n. 2 [289, n. 53], ${ }^{49}$ es usado también por Levi, que lo cita en $O C 2,72$ n. 2; en un caso similar al sucedido con el volumen de T. R. E. Holmes de 1928.

Levi usa con frecuencia en los dos volúmenes de su monografía, caso de este fragmento $O C, 2,71$, los seis volúmenes de una magna obra que, con el título de Geschichte Roms, W. Drumann y P. Groebe ${ }^{50}$ editaron en su segunda edición en Leipzig en un amplio intervalo de treinta años. Esta obra, sin caer en la apología mística de la figura de Augusto tan de moda en la época, usa las fuentes masivamente y con una rigurosa perspectiva filológica. Ambos autores subrayan que Octaviano no fue menos cruel que Antonio, y también que el hijo adoptivo de César tuvo que compartir los laureles de algunas victorias militares decisivas de su carrera con otros viri militares. Por último, la obra muestra cómo ciertos episodios bélicos fueron vitales a la postre para la conquista del poder único por el futuro Augusto.

A comienzos de la primavera de 37 (ref. 3 y 4), Antonio partió desde el Este hacia Italia con una fuerza naval de 300 barcos. La ciudad de Brindisi, y su acogedora bahía natural, rehusó permitir el fondeo de la flota antoniana. ${ }^{51}$ Éste puso entonces rumbo hacia Tarento y Octaviano viajó hasta allí para encontrarse con su colega. Comenzaron entonces las conversaciones que fructificarían en el tratado de Tarento.

47 Vale la pena citar el singular comentario de Syme acerca de este asunto en su temprana y positiva reseña a la obra de Levi: "To a modern reader the proscriptions form a distasteful chapter of history. It is easy to condemn them [las proscripciones]-and he who seeks to justify them may incur the charge of making divine Klio 'procuress to the Lords of Hell' [una oblicua mención al concepto de culpa, en inglés "sin", usando una eruditísima referencia por parte de Syme a Alfred Tennyson (In Memoriam, Sección 53). Agradezco el dato a A. R. Birley]. They must be understood in their context" (Syme 1934, 78).

48 Syme 1934, 78. Es sintomática esa mención de nuevo a la obra de T. R. E. Holmes.

49 BLuMENTHAL 1914.

50 DrumanN-Groebe 1899-1929.

51 Plu. Ant. 35.1. 
Las negociaciones entre ambos fueron lentas y hacia finales de julio o agosto se alcanzó un acuerdo, eso sí, con muchas dificultades. Para empezar, no estaba nada claro que Antonio fuera a apoyar a Octaviano en contra de los embates de Sexto Pompeyo. Las fuentes nos aseguran que la mediación de Octavia resultó crucial. ${ }^{52}$ Probablemente la dama tuvo un cierto protagonismo. Quizá Plutarco haya mejorado o embellecido el papel que Octavia jugó en la coyuntura, pero en nuestra opinión ${ }^{53}$ no mucho más de lo que en realidad debió de ocurrir. La mujer de Antonio y hermana de Octaviano sin duda influyó en la consecución de un acuerdo y, creemos, que el papel más claro de Octavia en Tarento fue el ejercer como arbiter entre su hermano y su esposo. ${ }^{54}$ El propio Syme, como vemos, es un tanto escéptico con respecto a esta idea. ${ }^{55}$

La otra cuestión importante debatida en Tarento (ref. 4), fue la renovación de los poderes triunvirales. El triunvirato había finalizado a finales de 38, dejando a sus miembros en una situación constitucional cuando menos discutible y confusa. Este aspecto formal no importaba quizá demasiado puesto que la realidad de dónde se encontraba el origen del poder estaba clara para todos. Ciertos hechos recientes de 40 y 39, como la muerte de Salvidieno Rufo a fines de 40 o el tratado de Miseno en la primavera de $39,{ }^{56}$ convencieron a Antonio y a Octaviano sobre la necesidad de definir su posición con mayor claridad. En Tarento el triunvirato se renovó formalmente para otros cinco años, finalizando probablemente el último día del año 33. ${ }^{57}$ Unas fechas más tarde, esta disposición fue ratificada por el pueblo de Roma.

Tarento no fue para Levi sino un episodio en la "lunga lotta sempre indiretta, mai dichiarata, fra Antonio e Ottaviano per il primato in Italia" (OC 2,73). Y sigue apuntado $(2,74)$ cómo Brindisi, Miseno y Tarento no pueden considerarse solo pausas en el conflicto por la supremacía, sino que pueden analizarse como tres episodios de la lucha de Octaviano por liberarse de la sujeción que Antonio pretendió siempre imponerle.

Antes mencionábamos que de las diez referencias cruzadas que existen a $O C$ en $R R$, seis se centran en los capítulos 19 y 20 de la obra de Syme. Es como si la influencia que la monografía de Levi posee en $R R$ se acentuara todavía más si cabe durante la escritura de esos dos capítulos que narran grosso modo los hechos del período de los años 40 a 30. Es muy difícil no estar de acuerdo con la idea de que Syme tenía a su lado el $O C$ cuando escribió los dos capítulos mencionados.

Antonio, retrasado en el Este en sus preparativos bélicos, recibió a comienzos de 35 la noticia de que su mujer se había presentado en Atenas inopinadamente. Octavia no venía sola. ${ }^{58}$ Traía consigo 2.000 soldados de élite y unos 70 barcos. Pero lo cierto es que Octaviano debía a Antonio mucho más que eso. Como apunta Syme: "The faithless colleague sent seventy ships: of ships Antonius had no need" ( $R R 265$ [336] -ref. 6-).

App. BC 5.93-95; D.C. 48.54 .3 y, en especial, Plu. Ant. 35.

Y aquí seguimos una línea que parte desde Blumenthal 1914, 84 y ss., hasta Pelling 1996, 26.

Vid. Vivas García 2013, 80 y ss.

Contra GABBA 1956, 203, n. 3; que matiza bastante la opinión de Syme otorgando credibilidad a la propia iniciativa de Octavia.

56 Recordamos que la muerte de Salvidieno fue aprobada mediante un senatus consultum ultimum y que en Miseno se dieron a conocer los nombres de quienes ocuparían la más alta magistratura del Estado hasta el 32.

57 Véase Pelling 1996, 67-68, para un magnífico estado de la cuestión sobre el sempiterno debate de si el triunvirato expiraba formalmente el 31 de diciembre de 33 o en la misma fecha pero al año siguiente.

58 App. BC 5.138. 
Compárese con lo escrito por Levi seis años antes: a Antonio "gli occorrevano truppe e non navi" (OC 2,134). Véase que Syme usa en inglés el mismo verbo que Levi había empleado en italiano, pero como sustantivo: ¡el verbo "necesitar"! Syme ha tomado prestada literalmente la misma expresión.

Antonio se enfrentaba a una disyuntiva crucial. Se limitó a aceptar las tropas que su esposa le ofrecía y le hizo permanecer en Atenas obligándola, eventualmente, a retornar a Roma. ${ }^{59}$

Levi piensa que todo el asunto de la afrenta a Octavia por parte de Antonio, impidiéndole reunirse con él en Siria, se exageró enormemente en las fuentes, al objeto de vincularlo con los celos de Cleopatra ante la legítima mujer romana de Antonio y, sobre todo, para ahondar en el topos de la decadencia moral del general romano en su abyecta y prohibida pasión por la reina de Egipto $(O C 2,134$, n.1). Syme sigue en esta ocasión, como en tantas otras, la opinión de Levi como la explicación más fiable $\left(R R 265\right.$, n. $3[336$, n. 27] $) .{ }^{60}$

El asunto del primer encuentro entre Cleopatra y Antonio en Tarso de Cilicia a finales de verano de 41 ha hecho correr muchos ríos de tinta.

En relación con este asunto, hemos agrupado las ref. 8 y 9 en $R R$ a la obra de Levi. En ambas se hace mención a la posibilidad de que Antonio y Cleopatra hubiesen contraído matrimonio en algún momento de su cohabitación, durante su estancia en Antioquía en el invierno de 37 a 36, como pretende J. Kromayer, ${ }^{61}$ o en todo caso antes del divorcio oficial entre Antonio y su legítima esposa romana a mediados de 32.

Ambos autores coinciden de nuevo en esta referencia y en otra anterior (ref. 5), en el uso de la bibliografía disponible. Ambos usan el trabajo pionero de U. Kahrstedt $(O C 2,122)^{62}$ y el fenomenal e importante trabajo de Johannes Kromayer de $1894 .{ }^{63}$

El autor de $R R$ se alinea con la tesis de Levi acerca de que tal matrimonio nunca tuvo lugar. Holmes es también de la misma opinión. ${ }^{64}$ De hecho, ésta ha llegado a ser la postura mayoritaria entre los historiadores hasta prácticamente la actualidad. ${ }^{65}$ Porque además, como Syme indica acertadamente en $R R 280$ n. 3 [355 n. 21] -ref. 9-, Antonio, como ciudadano romano, en ningún caso podía contraer matrimonio legalmente válido, según la ley romana, con una mujer extranjera.

Sea como fuere, a mediados de 32 Antonio abandonó a su esposa. Todo el episodio "fuelled Octavian's attacks and alienated valuable Italian support" ${ }^{66}$ El resto de la historia es conocido por todos.

\section{La influencia de $O C$ en $R R$ en el uso de la terminología política}

La influencia que $O C$ tuvo en el proceso de redacción de $R R$ se pone de relieve también en otro aspecto. Levi emplea en multitud de ocasiones a lo largo de los dos volúmenes de su obra los términos "partito" y "fazione" que aparecen, por ejemplo,

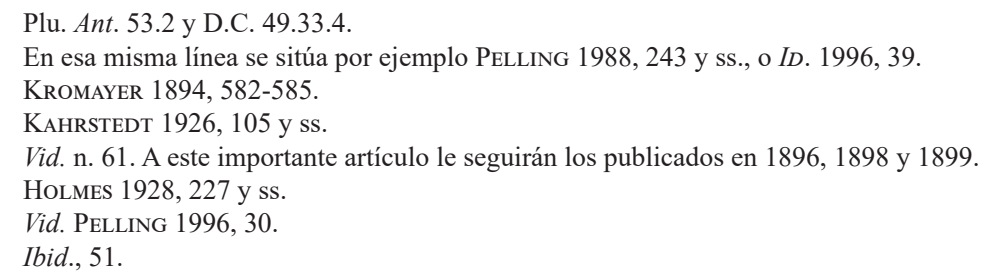


en $O C 1,229$ y ss. Levi usa asimismo, aunque en menor medida, otras expresiones como "fazione armata" o "nuovo stato". En concreto, las menciones de las palabras "partito" y "fazione", son muy numerosas. ${ }^{67}$

Por su parte, Syme emplea en numerosas ocasiones a lo largo de las 580 páginas de su libro los términos "party" y "faction"; y también "New State" que son, claramente, préstamos literales de la obra de Levi. ${ }^{68}$

Syme usa a lo largo de su libro expresiones de la época en la que escribe su obra, ya que una de sus características como historiador era usar términos contemporáneos a la hora de escribir para reflejar la realidad de la política romana tardorrepublicana. Esta característica suya como escritor le liga directamente, a nuestro modo de ver, con Levi, otro historiador que también empleaba con frecuencia expresiones tomadas de la política italiana de los años treinta del siglo XX para hacer referencia a las instituciones de la antigua Roma.

Syme usa así con frecuencia, a lo largo de $R R$, expresiones tales como "Free State" o "New Order", ambas procedentes de la politología moderna y que le sirven para identificar y nombrar realidades políticas del pasado; o bien usa una expresión como "Commonwealth" con relativa frecuencia. ${ }^{69}$ Este vocablo sirve a Syme para actualizar esa preocupación por la decadencia de la tradicional presencia itálica ante la mezcla con extranjeros y la pérdida generalizada de valores. ${ }^{70}$ Esa presencia itálica

${ }_{67}$ El vocablo "partito" se encuentra en $O C$ en el vol. I, páginas 11, 14, 15, 20, 24, 29, 30, 36, 37, 42, 43, 44, 47, $50,51,53,55,61,63,65,67,69,70,85,94,96,97,98,99,101,103,104,105,106,107,108,109,112,113$, $114,116,118,121,122,125,128,141,142,143,144,151,153,154,156,161,163,164,165,167,175,176$, $179,182,183,185,186,187,190,191,198,199,202,203,205,209,213,216,217,219,220,222,224,225$, $226,227,228,229,230,236,241,245,246,247,249,253,254,255,257,258,260$ y 261 ; y en el vol. II, en las páginas $2,3,5,6,7,18,19,20,22,25,28,35,45,51,52,53,92,94,142,145,148,155,161$ y 196 . El término "fazione" aparece en el vol. I, páginas: 59, 74, 92, 101, 114, 116, 142, 147, 153, 161, 163, 172, 180, 183, 184, $190,196,197,199,220,228,230,231,232,233,239,241,242$ y 251. Mientras que en el vol. II figura en las páginas $2,16,17,29,53,55,57,74,76,92,97,153,161$ y 186 . "Fazione armata" aparece en vol. I en las páginas 142 y 232, y en el vol. II en las 186 y 188; mientras que "nuovo stato" se cita en el vol. I, en las páginas 231,232 y 260.

68 En $R R$ el vocablo "party", traducción inglesa del término italiano "partito" se encuentra en las páginas: vii, viii (donde figura la expresión "party-leader"), x, 1, 4, 7, 9, 11, 12, 16, 17, 20, 23, 41, 44, 45, 47, 51, 55, 59, 60, 61, $62,65,68,69,73,78,86,88,94,95,96,98,99,106,108,109,114,120,121,127,130,137,141,152,153,154$, $155,156,160,161,166,167,170,173,175,182,187,188,189,191,193,194,197,198,199,208,214,217$, $220,221,222,226,227,229,234,236,239,241,242,263,267,268,270,280,281,282,288,289,291,292$, $303,307,311,316,323,325,331,334,336,340$ (vale la pena reflejar la frase: “Augustus' character remains elusive (...) He was no puppet (...) his unique primacy must not obscure the reality from which it arose -the fact that he was the leader of a party -capoparte-"), 341, 343, 346, 347, 349, 351, 365, 368, 376, 383, 409, 413, 419, 423, 424, 438, 444, 453, 479, 490, 495, 509, 513, 518, 521 y 524. “Faction” está presente en las páginas: 4, 7, 11, 17, 19, 22 (donde escribe sobre el grupo aristocrático: "They called themselves Optimates: they might properly be described, in contemporary definition, as a faction or gang"), 26, 43, 46, 49, 51, 55, 56, 60, 65, 67, $72,75,88,89,97,99,100,107,109,113,114,120,127,129,130,132,133,154,157,163,164,169,180$, $181,191,198,209,220,237,239,250,281,285,288,291,349,350,359,360,365,368,384,417,422,423$, 427, 459, 491, 492, 493, 510, 523 y 524. La expresión "New State", muy del gusto de Levi, puede leerse en las páginas: $7,38,320,321,342,344,350,353,360,375,397,416,418,440,445,450,451,453,460,461,466$, $469,478,479,484,490,498,506,509,512,513,514,517$ y 521 . Una variante de esta expresión es el término "Novus Status", presente en las páginas: 2, 320 y 324.

69 Hemos detectado un interés de Syme por elegir palabras con presencia en la Constitución no escrita del Reino Unido. En efecto, la expresión "Estado libre" ("Free State") se observa en $R R$ en: 1, 8, 10, 13, 36, 43, 50, 53, 59, 94, 205, 234, 245, 257, 315, 317, 349, 373, 376, 407, 408, 441, 492, 496, 512, 513 y 515. "New Order" puede leerse en: 3, 243, 257, 321, 328, 335, 351, 374, 459 y 479. Por último, “Commonwealth" figura en: 39, 42, 43, $57,78,105,131,136,143,145,158,159,319,331,352,407,518$ y 520 .

70 Cf. WulfF 2014, 20. 
tradicional, que Wulff denomina "raza imperial", ${ }^{71}$ es la que debe soportar las cargas impositivas y de efectivos que requiere el Imperio que ahora se establecerá.

No deja de ser sorprendente el uso que Syme que hace, por ejemplo, del vocablo "constitutional" al comienzo y al final de su obra: así en $R R 1$ [17] y en 518 [648]. Por tanto, los términos usados por Syme, tomados de Levi, son reveladores. El concepto de "Nuevo Estado", "Nuovo Stato", "New State", "New Order", era muy del gusto de la terminología fascista europea, en especial de la mussoliniana, de la que tanto bebió la obra de Levi. ${ }^{72}$ Como tampoco es casual que los títulos de sendos capítulos de $R R$ evoquen las distintas "marchas sobre Roma" (capítulos IX y XIII) o que otro lo encabece el sintomático título de "Dux" (capítulo XXI). ${ }^{73}$

Este gusto de Syme por usar términos contemporáneos en sus escritos para referirse a realidades de la Roma antigua lo hemos catalogado en fecha tan temprana como 1931. En una reseña publicada ese año en The Classical Review sobre un libro del profesor alemán Walther Rehm (1901-1963) cuyo tema era la caída del Imperio Romano de Occidente en el pensamiento occidental, ${ }^{74}$ Syme asegura que el problema de la caída del Imperio Romano poseía seguramente rasgos mucho más contemporáneos de lo que se pensaba en su época. Se trata de una velada referencia por su parte a la caída de determinados Estados o Imperios contemporáneos. Prosigue haciendo una mención muy oblicua a las teorías totalitarias del fascismo y del nazismo al escribir "(...) to mention only theories about racial mixture (...)". ${ }^{75}$ Y continúa con una referencia más clara si cabe a la Revolución Rusa de 1917 que demolió el gobierno de los zares: “(...) and about the destruction of the bourgeoisie and civilisation by a Red Army of peasants". ${ }^{76}$

Dos años más tarde, en 1933, y en un artículo titulado "Some Notes on the Legions under Augustus", ${ }^{77}$ un trabajo muy técnico de historia militar, Syme escribe que la seguridad política y militar del imperio y la "peace of the world", pasaba por una agresiva política de conquistas. Para asegurar la estabilidad del Estado romano, era urgente contar con unas fronteras fuertes y adecuadas. Era imprescindible la anexión de los Alpes y el Ilírico, y ya puestos también de la Germania. Según la visión de Syme: "This was a plan of long date, meditated and well matured and not carried out before Spain had been pacified by arms, the East by diplomacy - and before the New Army was ready and at its best". ${ }^{78}$ Se trata de una referencia al "nuevo ejército" de reclutas voluntarios, al menos en el proyecto inicial, implementado a finales de julio de 1914 por el general Horatio Herbert Kitchener (1850-1916), Secretario de Estado de Guerra del gobierno británico, en relación con el comienzo de las hostilidades de la Primera Guerra Mundial. Otra muestra más del gusto de Syme por usar expresiones históricas contemporáneas para referirse a hechos de la Antigüedad, en este caso al nuevo ordenamiento del ejército legionario romano en época augústea.

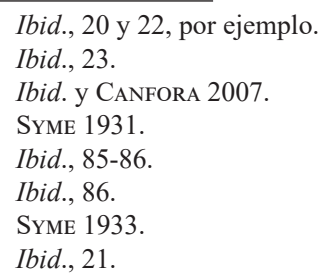




\section{Conclusión}

Levi narra en su obra, en clave política y sociológica, la crónica del ascenso al poder supremo de un "jefe de partido"-"capoparte"- que perpetra un golpe de estado contra las instituciones exánimes y periclitadas de una forma de gobierno consumida por el desgaste, la corrupción y su propia idiosincracia. Este joven caudillo, cuyos inicios son los de un aventurero, crea un nuevo "partido" -"partito", "party"- con elementos provenientes de toda Italia: un partido único y gigantesco de corte tradicional y conservador. Un partido que se aglutina y se reconoce por el consenso de toda la península -“tota Italia"- alrededor de ese líder carismático mediante un juramento de fidelidad. Un líder al que todos siguen ciegamente cual soldados a su imperator, puesto que se identifican con su firme mano de gobierno, recta y magistral. Asistimos a un programa de regeneración en todos los órdenes. Un auténtico "back to basics".

Un partido único se constituye en Gobierno, en un Nuevo Estado -"Nuovo Stato", "New State", "New Order"-.${ }^{79}$ Un "stato nuovo" que da cuerpo a un nuevo y promisorio orden político. Una nueva "aurea aetas" que auspicia una renovación total y que pretende, al menos nominalmente, finiquitar la vieja política en un proceso que muy bien puede tildarse de una auténtica "Revolución Romana". ${ }^{80}$

¿No puede considerarse lo anterior un excelente resumen de la obra más conocida de Syme? Este mismo autor acaba $R R$ sentenciando: "Dux had become Princeps and had converted a party into a government. For power he had sacrificed everything; he had achieved the height of all mortal ambition and in his ambition he had saved and regenerated the Roman People" (RR 524 [655]). ¡Qué mejor resumen en tres breves líneas de los cientos de páginas precedentes!

A lo largo de las páginas anteriores hemos tratado de desbrozar el influjo que la obra del historiador italiano Levi, prácticamente desconocida en el Reino Unido en la época en que Syme la estaba utilizando, tuvo en el proceso de redacción y composición de la obra más conocida de Syme.

Hasta ahora estas concomitancias habían pasado prácticamente desapercibidas. ${ }^{81}$

Pero las diferencias entre ambas obras nos hablan tanto de las mismas como sus semejanzas. En su análisis de 1940, Momigliano acusaba ya a ambos autores de reducir el proceso que narraban a una mera "struggle of factions". ${ }^{82}$ Para Levi la investigación prosopográfica, el modelo iniciado por Münzer y llevado a sus últimas consecuencias por Syme, no es solo insuficiente, sino que proporciona una imagen deformada de la realidad ya que tiende a sobrevalorar indebidamente las ambiciones, sentimientos y resentimientos de los grupos privilegiados. Los ideales, la ideología que mueve a estos grupos, son considerados como algo superfluo, instrumental en la visión de Syme. Levi disiente de esta idea.

A diferencia del neozelandés, para Levi la etapa de guerras civiles con la que finaliza la República no es solamente "scontro di fazioni", ${ }^{83}$ sino también un período de movimientos de masas de clientelas, de marcado carácter militar, que iban más allá de las divisiones geográficas del imperio ordenadas por los triunviros. La

\footnotetext{
79 Incluso podríamos hablar aquí cínicamente de "Free State".

80 Cf. WulfF 2014, 24, comentando $R R$.

81 Cf. parcialmente, para la influencia de Levi en el uso del término "partido": LóPez BARJA DE QuIROGA 2016, 38.

82 Momigliano 1940, 78.

83 GARA - FORABOSCHI 1993, 16.
} 
composición de las clases dominantes quedaría así modificada y puesta en cuestión, durante una o más generaciones. Se puede convenir en que para Levi esta fue la auténtica "Revolución Romana". En Levi es fundamental la cuestión de las dinámicas internas de las clases sociales dirigentes y sus interrelaciones. Casi una cuestión "à la Brunt". En Syme no vemos nada similar.

Podemos afirmar lo anterior a pesar de que en ambos autores la narración de la transición de "Ottaviano Capoparte" a "Augusto príncipe" posee muchos puntos de contacto, como hemos podido comprobar en las páginas anteriores. Y es que el "spell" de Levi sobre Syme comenzó en fecha tan temprana como 1934, fecha de la publicación de la reseña del docente de Trinity al Ottaviano Capoparte. ${ }^{84}$ En esta reseña, a excepción de la siguiente frase: "We sometimes suspect that [en $O C$ ] adequate justice has not been done to $\mathrm{M}$. Antonius", ${ }^{85}$ existen muy pocas críticas por parte de Syme al tratamiento que efectúa Levi del período en la totalidad de los dos volúmenes.

Si hemos conseguido suscitar algún debate o provocar la curiosidad sobre este apasionante y complejo tema nos sentiríamos razonablemente satisfechos. Esperamos haber convencido de que existe una gran influencia del Ottaviano Capoparte de Levi en la Roman Revolution de Syme a todos los que se hayan tomado la molestia de leer hasta este último párrafo.

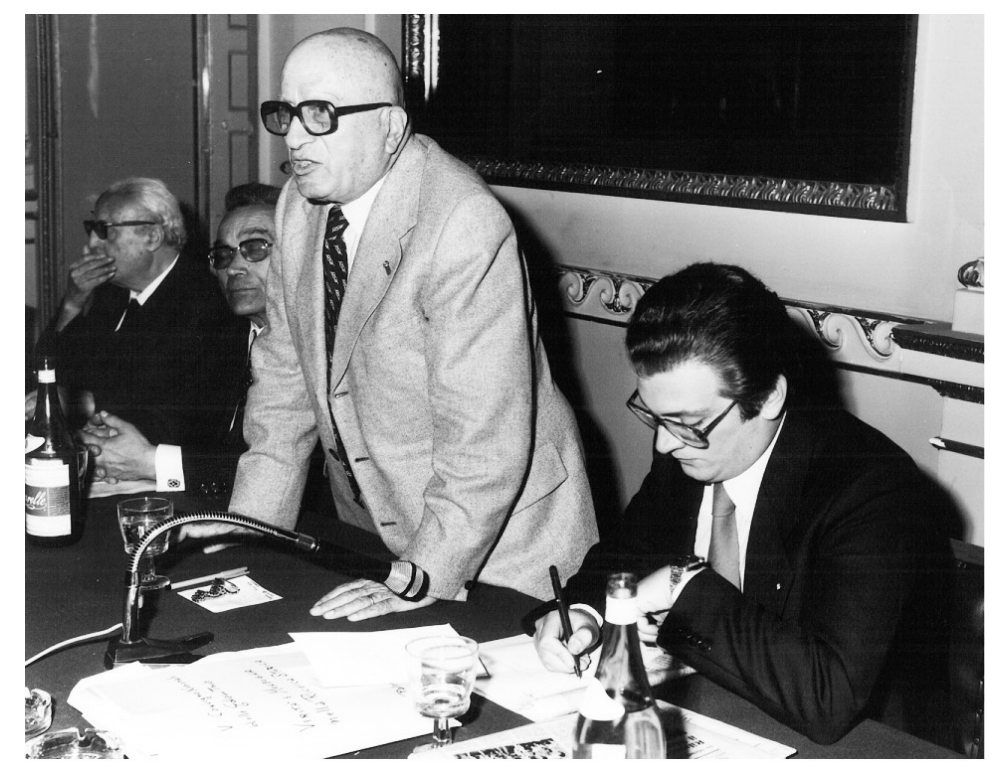

Figura 4. M. A. Levi, Mayo de 1980. De izquierda a derecha: P. Gerbore, C. Vassillakis, M. A. Levi y T. Romano. Coloquio auspiciado por la editorial Thule. Palermo.

\footnotetext{
${ }_{84}$ Obra que desde el principio de la reseña califica de: “(...) a work of solid erudition and permanent value” (SYME 1934, 76).

$85 \quad$ Ibid., 77.
} 


\section{Referencias bibliográficas}

ALFÖLDY, G.

(1983): “Sir Ronald Syme, 'Die römische Revolution' und die deutsche Althistorie”, Sitz. der Heidelberger Akademie der Wissenschaften, Philolsophisch-historische Klasse 1, 5-42. (2015): "The crisis of the third century from Michael Rostovtzeff and Andrew Alföldi to recent discussions", [en] J. H. Richardson - F. Santangelo (eds.), Andreas Alföldi in the Twenty-First Century (=HABES 56), Stuttgart, 201-217.

Alonso Troncoso, V. (1990): "Desesperadamente ajeno: Sir Ronald Syme and The Roman Revolution", Estudios Clásicos 97, 41-64.

Arce Martínez, J.

(1990): "De triunfador a dictador: la peculiar revolución de Octavio César Augusto", Claves de Razón Práctica 6, 48-53.

(1994): "Sir Ronald Syme: la historia romana", Revista de Occidente 152, 37-48.

(2010): "Prólogo", [en] Syme, 2010, vii-xviii.

BIRLEY, A. R.

(1989): "Sir Ronald Syme", The Independent, September 7.

(2016): "A Letter from Momigliano to Syme, May 1967", Politica Antica 6, 151-164.

Blumenthal, F. (1914): “Die Autobiographie des Augustus", Wiener Studien 36, 84-103.

BOWERSOCK, G.

(1980): "The Emperor of Roman History", The New York Review of Books, $6^{\text {th }}$ March, 8-13. (1991): "Ronald Syme (March 11, 1903-September 4, 1989)", Proceedings of the American Philosophical Society 135, 118-122.

(1994): "Ronald Syme 1903-1989", Proceedings of the British Academy 84, 539-563.

(1995): "Ronald Syme 1903-1989", The Brazen Nose 29, 48-67.

Bravo, G. (1989): Poder político y desarrollo social en la Roma antigua, Madrid.

BRUNT, P.

(1989): "Sir Ronald Syme", The Times, September 6 [add. M. T. Griffin, The Times, September 13, 1989].

(1996): "Nota sobre el obituario de Sir Ronald Syme escrito por G. Bowersock", The Brazen Nose 30, 43-44.

Caballos Rufino, A. (1993): “Introducción”, [en] R. Syme, Élites Coloniales. Roma, España y las Américas, Málaga, 9-18 (trad. A. Caballos Rufino).

CANFORA, L. (2007): La prima marcia su Roma, Roma-Bari.

Cannistraro, Ph. V. (1975): La fabbrica del consenso: fascismo e mass media, Roma-Bari.

Christ, K. (1990): "Ronald Syme”, Neue Profile der Alten Geschichte, Darmstadt, 188-247.

DAhlheim, W. (2003): "Ronald Syme. Geschichte als aristokratische Gelehrsamkeit und literarische Kunst", [en] Ch. Selzer - U. Walter (eds.), R. Syme, Die römische Revolution: Machtkämpfe im antiken Rom, Stuttgart, 713-731 (trad. F. W. Eschweiler y H. G. Degen).

Devine, A. M.

(1989a): “Sir Ronald Syme (1903-1989): A Roman Post Mortem”, AncW 20, 67-75.

(1989b): "Sir Ronald Syme and The Roman Revolution", AncW 20, 77-92.

(2004): "Syme, Ronald (1903-89; kt 1959)", [en] R. B. Todd (ed.), The Dictionary of British Classicists, Bristol, 3941-3945.

Di Donato, R. (1995): "Materiali per una biografia intellettuale di Arnaldo Momigliano, 1. Libertà e pace nel mondo antico", Athenaeum 83, 213-244.

Drumann, K. W. - Groebe, P. (1899-1929²): Geschichte Roms in seinem Übergang von der republikanischen zur monarchischen Verfassung. I-VI, Berlin-Leipzig. 
GABBA, E. (1956): Appiano e la storia delle guerre civile, Firenze.

GAlsterer, H. (1990): “A Man, a Book and a Method: Sir Ronald Syme's Roman Revolution after Fifty Years”, [en] K. A. Raaflaub - M. Toher (eds.), Between Republic and Empire. Interpretations of Augustus and his Principate, Berkeley, 1-20.

GARA, A. - Foraboschi, D. (EDS.), (1993): "Introduzione", [en] Il triumvirato costituente alla fine della Repubblica romana. Scritti in onore di Mario Attilio Levi (=Bibliotheca di Athenaeum 20), Como, 7-20.

VIVAs GARCíA, G. A.

(2013): Octavia contra Cleopatra. El papel de la mujer en la propaganda política del Triunvirato (44-30 a.C.), Madrid.

(2016): Ronald Syme. El camino hasta La Revolución Romana (1928-1939), (=Col·ecciò Instrumenta 54), Barcelona.

GrifFIN, M. T. (1990): “Sir Ronald Syme, 1903-1989”, JRS 80, xi-xiv.

HinARD, F. (1985): Les proscriptions de la Rome républicaine (=Collection de l'École française de Rome 83), Rome.

Holmes, T. R. E.

(1923): The Roman republic and the founder of the empire, Oxford, 3 vols. (1928): The Architect of the Roman Empire. Volume 1, Oxford.

Kahrstedt, U. (1926): "Syrische Territorien in hellenisticher Zeit", Gött. Abh., phil. hist. Kl. $19 / 2$.

Kromayer, J. (1894): "Kleine Forschungen zur Geschichte des zweiten Triumvirats", Hermes 29/4, 556-585.

LEVI, M. A.

(1933): Ottaviano Capoparte. Storia politica di Roma durante le ultime lotte di supremazia, Firenze, 2 vols. (1951): Il tempo di Augusto, Firenze.

(1989): “Alla scuola di Gaetano De Sanctis negli Anni Venti”, Storia della Storiografia $16,3-14$.

LóPez BARJA De Quiroga, P. (2016): “Conflicto ideológico y guerra civil en la Tardía República: causa, memoria y miedo", [en] N. Ruiz de los Llanos - M. Rubio - C. Rieszer (comps.), Actas del XXIII Simposio Nacional de Estudios Clásicos. Ciudadanía y poder político en el mundo clásico, Salta, 33-48.

Marcone, A. (2015): "Recensione di La rivoluzione romana", Rivista Storica Italiana 127/2, 624-628.

Matthews, J. (2010): "Ronald Syme, Constantine the Great and the Second Roman Revolution", [en] Roman Perspectives. Studies in the social, political and cultural history of the First to Fifth Centuries, Swansea, 41-56.

Millar, F. (1981): “Style Abides”, JRS 71, 144-152 (http://dx.doi.org/10.2307/299503).

Momigliano, A.

(1940): "Review: Ronald Syme, The Roman Revolution. Oxford: The Clarendon Press, 1939. pp. 568 21s.”, JRS 30, 75-80 (http://dx.doi.org/10.2307/296948).

(1962): "Introduzione", [en] R. Syme, La Rivoluzione Romana, Roma, ix-xv (=Terzo Contributo alla storia degli studi classici e del mondo antico, II, Roma, 729-737).

Murray, O. (2010): "Momigliano on Peace and Liberty", Acta Universitatis Carolinae Philologica 1 (=Graecolatina Praguensia 23), 81-96.

Musti, D. (1993): “Levi, Mario Attilio”, Enciclopedia Italiana. V Appendice, Roma, App. III, I, 987. 
Pelling, C.

$\left(1996^{2}\right)$ : "The Triumviral Period", [en] A. K. Bowman - E. Champlin - A. Lintott (eds.), The Cambridge Ancient History, vol. 10. The Augustan Empire, 43 BC-AD 69, Cambridge, 1-69.

(2015): “The Rhetoric of The Roman Revolution", Syllecta Classica 26, 207-247 (http:// dx.doi.org/10.1353/syl.2015.0004).

Pelling, C. (ED.), (1988): Plutarch, Life of Antony, Cambridge.

Polverini, L. (2006): “Momigliano e De Sanctis", [en] L. Polverini (ed.), Arnaldo Momigliano nella storiografia del novecento, Roma, 11-35.

Rebenich, S. (2014): “Syme, Ronald”, [en] Brill's New Pauly Supplements I - Volume 6: History of classical Scholarship - A Biographical Dictionary, Leiden-Boston, 607-609.

Rotondi, G. (1912): Leges publicae populi romani, Milano.

Santangelo, F. (ED.), (2016): Approaching the Roman Revolution. Papers on Republican History by Sir Ronald Syme, Oxford.

Santarelli, E. (1991): “De Vecchi, Cesare Maria", [en] Dizionario Biografico degli Italiani, 39 , online.

SYME, R.

(1931): "The Decline and Fall. Der Untergang Roms im abendländischen Denken by Walter Rehm", The Classical Review 45/2, 85-86 (https://doi.org/10.1017/ S0009840X00055700).

(1933): "Some notes on the legions under Augustus", JRS 23, 14-33 (http://dx.doi. org/10.2307/297202).

(1934): "From Octavian to Augustus. Mario Attilio Levi, Ottaviano Capoparte", The Classical Review 48/2, 76-78. (=RP, VI, 1-5), (https://doi.org/10.1017/ S0009840X00064489).

(1937): "Augustus and Agripa. B. M. Allen, Augustus Caesar, and F. A. Wright, Marcus Agrippa, Organizer of Victory", The Classical Review 51/5, 194-195 (https://doi. org/10.1017/S0009840X00071626).

(1938): "Caesar, the Senate and Italy", Papers of the British School at Rome 14, 1-31 (=RP I, 88-119), (http://dx.doi.org/10.1017/S0068246200005833).

(1939): The Roman Revolution, Oxford [reimp. 2002; trad. española de A. Blanco Freijeiro, Madrid, 1989].

(1979): Roman Papers I-II, E. Badian (ed.), Oxford.

(1991): Roman Papers VI-VII, A.R. Birley (ed.), Oxford.

(2010): La Revolución Romana, Barcelona [prólogo de J. Arce Martínez].

Toher, M. (2009): “Tacitus' Syme”, [en] A. J. Woodman (ed.), The Cambridge Companion to Tacitus, Cambridge, 317-329.

TraInA, G. (2014): “Introduzione alla seconda edizione italiana”, [en] R. Syme, La rivoluzione romana, Torino, vii-xxii.

Walter, U. (2002): "Der Historiker in seiner Zeit: Ronald Syme und die Revolution des Augustus", [en] J. Spielvogel (ed.), Res Publica Reperta. Festschrift für Jochen Bleicken zum 75. Geburtstag, Stuttgart, 137-152.

Wiseman, T. P. (1998): “Late Syme. A Study in Historiography”, [en] T. P. Wiseman (ed.), Roman Drama and Roman History, Exeter, 135-152.

WulfF, F. (2014): "La "unidad de Italia" y el Augusto de Syme: nacionalismo, fascismo y élites en el período de entreguerras", inédito. 